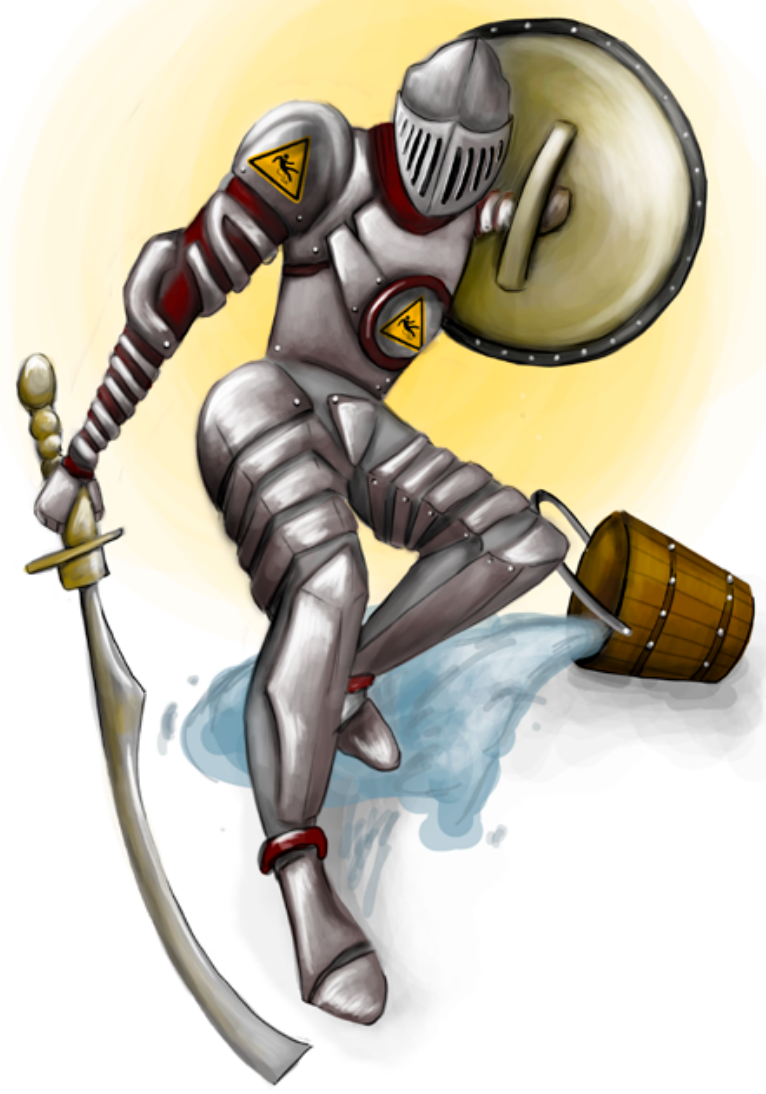

- Capítulo 1 -

\title{
Proyecto de un Laboratorio de Riesgos Laborales para la Institución Universitaria Politécnico Grancolombiano
}

\section{Autores}

Luis Martín Trujillo Flórez. Ingeniero electricista de la Universidad Nacional de Colombia. Máster en Edición Universitaria Universidad de Salamanca. Maestría en Ambientes Virtuales de Aprendizaje Universidad de Panamá. Especialista en virtualidad 
Centro de Altos Estudios de Argentina. Líder del Laboratorio de Experiencias Inmersivas de Aprendizaje Educación Virtual Politécnico Grancolombiano.

Correspondencia: mtrujilo@poligran.edu.co

Gisette Katherine Velandia Vargas. Psicóloga Especialista en Gerencia del Recurso Humano, Máster en Sistemas Integrados de Gestión, Actualmente coordinadora del programa de formación profesional en Gestión de la seguridad y la salud laboral. Consultora especialista en la gestión del talento humano e implementación de programas de riesgos psicosocial. Docente Universitaria.

Correspondencia:gkvelandia@poligran.edu.co

\section{Resumen}

El presente documento plasma el proceso del desarrollo del simulador denominado Laboratorio de Riesgos Laborales para el programa de Gestión de la Seguridad y Salud Laboral el cual se ocupa de los riesgos Biológico, Ergonómico, Físico, Químico, Natural y Seguridad para cinco tipos de empresas diferentes (laboratorio, manufactura, oficina, pozo petrolero y empresa de servicios generales). Se tratará cómo se planeó la estrategia pedagógica y cómo se realizó el diseño instruccional para la creación de la herramienta de software a través de una planeación que va desde la descomposición de la competencia, pasando por un diagrama de tareas y finalizando en la elaboración del guion, para de allí pasar a diseño gráfico, diagramación y programación. El proyecto finaliza con la publicación de la herramienta en las aulas virtuales para los estudiantes del programa en dicha modalidad.

\section{Palabras claves}

Laboratorio online, simulador, riesgos laborales, análisis de riesgos.

\section{Introducción}

El programa profesional de Gestión de la Seguridad y Salud Laboral es uno de los más recientes en la Institución Universitaria Politécnico Grancolombiano, aun así, cuenta con una población estudiantil cercana a los cuatro mil estudiantes. 
Para todo el equipo académico significa un reto que cada vez es más exigente, donde se propende una educación de alta calidad y un currículo acorde con las necesidades del entorno laboral en pro de los estudiantes. Conscientes de este compromiso el equipo académico ha desarrollado diversas estrategias educativas, ha adquirido algunos softwares de simulación y otras herramientas de apoyo académico, también está adelantando un rediseño de todos los materiales y módulos a través del proyecto denominado Escenarios para el aprendizaje que estructura las rutas que le permiten al estudiante afianzar sus conocimientos y apropiar sus competencias.

Dentro de esta propuesta se implementaron diversos módulos con una estructura teórico-práctica, en la cual los "escenarios" darán cuenta de la parte teórica; y con el uso de simuladores se desarrollará el área práctica que pretende evidenciar la adquisición de las competencias. Aquí se integran los dos proyectos, el de escenarios para el aprendizaje y el del laboratorio de riesgos laborales, pues los escenarios trabajarán la parte teórica y el laboratorio dará cuenta de la parte práctica, integrado en un módulo. Sin embargo, el proyecto es más ambicioso porque reúne toda la línea de Seguridad e Higiene Laboral, es decir, el estudiante trabaja todo el laboratorio en tres semestres de su programa curricular.

El proyecto nace intentado cubrir la necesidad de evidenciar los conocimientos adquiridos por parte de los estudiantes que salen a enfrentarse a contextos reales, por eso la estrategia procura ser transversal en varios módulos del programa, crear escenarios en los que los estudiantes puedan confrontarse con situaciones próximas a la realidad. De igual manera, se pretende apoyar la práctica aplicada que hacen los alumnos al final del programa en una empresa real. También se constituye como insumo para la construcción de un futuro consultorio empresarial virtual en gestión de la seguridad y salud laboral, con la ventaja de ser uno de los primeros del país.

El proyecto es una propuesta que se generó en el Departamento Académico de Psicología de la Facultad de Cultura, Sociedad y Creatividad en conjunto con Educación Virtual, fue estructurado, diseñado y desarrollado por varios tutores de la Institución y el direccionamiento fue hecho por el Laboratorio de Experiencias Inmersivas de Aprendizaje de Educación Virtual, por tal razón, contó con asesoramiento en el diseño y modelamiento de los seis (6) 
expertos en el análisis de riesgos laborales quienes contribuyeron con sus conocimientos para plasmarlo en los guiones, y en una metodología de análisis para los diferentes tipos de riesgo. El diseño y la programación fue tercerizada a la empresa Picolab, quien dispuso al servicio de este un equipo de ingenieros, animadores, diseñadores gráficos y desarrolladores. Aunque la institución tiene un área interna de desarrollo de software educativo, la envergadura del proyecto requirió tercerizar estos servicios.

Este macroproyecto de un laboratorio de riesgos laborales que en su interior cuenta con un simulador para cada riesgo laboral (biológico, ergonómico, físico, químico, natural, seguridad), es decir, seis simuladores, y se recrea en cinco empresas diferentes (laboratorio químico, manufactura, oficina, pozo petrolero, empresa de servicios), implica la realización de treinta simuladores, puesto que cada riesgo y cada empresa tiene sus individualidades y características propias que hacen que los guiones, diseño y desarrollo se realizaran para cada uno de ellos; más de cien animaciones en 2D, más de cincuenta escenarios en 3D, cerca de 130 minutos de grabación de audios, más de treinta personajes, un avatar, mil quinientas páginas de guiones, y un trabajo arduo de casi dos años, vuelven este proyecto significativo para la Institución que apuesta por un desarrollo in house del software.

Este libro cuenta todo el proceso de elaboración, desde la maquinación hasta la elaboración y publicación en las aulas, y deja la gran enseñanza la posibilidad de generar nuevos proyectos que permitan solventar las necesidades académicas de los diferentes programas académicos.

\section{Marco Teórico}

\section{Antecedentes}

Desde el inicio del programa Gestión de la Seguridad y Salud Laboral fue detectada la necesidad de contar con laboratorios de práctica en los que los estudiantes evidenciaran en un contexto determinado sus habilidades y comportamientos al trabajar una situación, por tal razón, lo primero 
que se contempló fue contratar un laboratorio virtual puesto que es una representación informática de los laboratorios tradicionales, que permiten realizar experimentos, investigaciones, prácticas académicas y científicas, generando procesos muy similares a los que existen en el contexto real porque apoyan y promueven el aprendizaje de los estudiantes y aumentan las opciones de experimentos (Pascuas, Bocanegra, Ortiz y Pérez, 2012)

Como se verá en los siguientes capítulos se hizo un análisis de algunas herramientas de software disponibles en el mercado que permitieran el análisis de riesgos laborales. La gran dificultad fue encontrar una herramienta que cubriera los riesgos contemplados por la Guía Técnica Colombiana - GTC 45 (2012) la cual define los siguientes tipos de riesgo laboral: Riesgo Biológico, Ergonómico o Biomecánico, Físico, Químico, Natural y Seguridad. La indagación arrojó como resultado que hay varios softwares de riesgos laborales pero la mayoría sólo comprenden los riesgos de seguridad, algunos incluyen los químicos y otros, en menor medida, los físicos. Pero no existe una herramienta que integre los seis tipos de riesgos. También se encontró que en muchos casos el proceso está más centrado a la identificación de los riesgos y que acciones realizar para que no ocurra el suceso. No se halló una herramienta que hiciera el proceso de identificación, diagnóstico, evaluación, análisis y mitigación de este, que son los pasos que propone la guía técnica, incluso la guía va más allá porque plantea un proceso de seguimiento e implementación de los cambios en la empresa.

Se encontraron algunas herramientas que permiten $y$ ayudan al diligenciamiento de la matriz de peligros que resultan útiles para el proceso de aprendizaje, pero presentan dos inconvenientes: el primero, no generan un caso de estudio, por consiguiente, el estudiante debe contar con una empresa para realizar el proceso; el segundo, que sólo aplica la GTC 45. Sin embargo, de acuerdo con el riesgo se encontró que hay otras metodologías más apropiadas para la evaluación de los mismos, por ejemplo, Riesgo Biológico (Biogaval), Ergonómico o Biomecánico (RULA, REBA, OWAS), Físico (GTC-45), Químico (Gatiso de Benceno), Natural (Metodología de colores) y Seguridad (GTC-45). Estas metodologías fueron las que se aplicaron en el simulador, cada una de ellas se adaptó para que el estudiante pudiera hacer un análisis más estructurado y más cercano a las necesidades del entorno.

\section{Problemática que atiende}


Una de las problemáticas presentes en los diferentes programas virtuales es la evidencia de la adquisición de las competencias por parte de los estudiantes. Según Tobón (2004) "Las competencias son procesos a través de los cuales resuelven problemas del contexto laboral articulando el saber hacer, el saber conocer y el saber ser". Por tal razón, se debe evidenciar que el estudiante puede integrar los conocimientos adquiridos, ponerlos en evidencia en un escenario real e integrarlos a sus actitudes y valores.

Dicha necesidad se evidencia en el programa académico Profesional de Seguridad y Salud Laboral, por lo tanto, se hace palpable la necesidad de generar espacios y escenarios de práctica que permitan evidenciar que los estudiantes son capaces de desenvolverse adecuadamente en un entorno laboral, aunque en la malla curricular ya existen algunos módulos de esencia práctica, el más significativo es la "práctica laboral" que la cursan los estudiantes de último semestre. Dicho proceso se trabaja con diarios de campo a través de reportes semanales de lo que hacen en la empresa y debe ser coherente con el proyecto que los estudiantes presentan como intervención en la empresa.

De acuerdo con el análisis hecho por los tutores a cargo de los últimos semestres, los estudiantes evidencian deficiencias cuando deben confrontar diversas situaciones reales debido a diferentes causas, la más notoria, es la falta de un acercamiento a escenarios reales que les permita afianzar y confrontar sus conocimientos teóricos en situaciones reales. Este mismo problema de no tener un acercamiento previo a la praxis que les permita un mejor enfoque hacia lo que va a ser su quehacer profesional y les garantice un desempeño adecuado en un contexto real, lo manifiestan los estudiantes. En otras palabras, tienen mucha conceptualización teórica con poca práctica.

Esta problemática de requerir escenarios de práctica tiene varias complejidades, pues no sólo es crear un espacio, sino que al ser un programa virtual exige necesariamente que sea a través de ambientes virtuales de aprendizaje porque no todas las personas tendrían la posibilidad de ir hasta su laboratorio si este fuera presencial. Para solucionar esta problemática se apuesta al diseño y desarrollo de un software educativo tipo laboratorio virtual que da cuenta de la parte práctica de los diferentes módulos, los estudiantes trabajan y simulan en línea. De acuerdo con Barrios y Marín (2013) “Utilizar un laboratorio remoto es una experiencia cercana a un uso real en un laboratorio presencial (casi idéntico), por lo que es capaz de sustituir a éste sin afectar negativamente la labor del usuario". 
Otra dificultad es la alineación entre la teoría y la práctica. Para ello, se integra en el módulo los contenidos con el simulador, es decir, el proceso del estudiante inicia con "escenarios de aprendizaje" los cuáles se centrarán en los conceptos, fundamentos del análisis de los riesgos laborales, la parte teórica. La parte práctica estará a cargo de la herramienta de simulación. Las estrategias implementadas para este proyecto se visualizan de una manera holística de tal forma que los contenidos del módulo como lecturas, objetos de aprendizaje, actividades están alineadas con el proceso de simulación, en su mayoría coinciden los temas del módulo con lo que se trabajan dentro del simulador.

¿Cómo lograr la sinergia entre una herramienta de software y los contenidos del módulo?, se puede hacer de dos maneras. La primera es obtener la herramienta y según esta construir y diseñar el módulo; la segunda consiste en diseñar la herramienta de simulación a partir del sílabo y las necesidades de aprendizaje, obviamente para aplicar esta estrategia debe contarse con un equipo de producción que tenga asesores pedagógicos, guionistas multimedia, diseñadores gráficos, animadores, ilustradores, desarrolladores de software e ingenieros de sistemas, sin contar todo el capital humano en la gestión y consecución de recursos para que sea viable. Esta última es la apuesta institucional para el desarrollo del proyecto.

Otra dificultad proviene de la particularidad de cada riesgo. El riesgo ergonómico depende de la observación y el análisis que se haga de cada persona, por lo tanto, llevar un proceso de este estilo a un escenario virtual no es nada fácil. Según Amaya (2009) La simulación computarizada es la representación digital de un sistema real, que mediante una serie de algoritmos preestablecidos responde a las características naturales de una parte de la realidad, es decir, el laboratorio sirve para la preparación del estudiante, para el mejoramiento de sus habilidades y la evidencia de sus competencias, pero no puede reemplazar lo que llegue a vivir la persona en su quehacer laboral. Lo anterior es un punto definitivo porque tanto un laboratorio tradicional o uno virtual son una representación de un fragmento de la realidad, por ejemplo, si revisamos un riesgo natural puede que se tenga todo lo que se requiere para la mitigación del mismo, pero en la ocurrencia de un desastre nada asegura que las personas reaccionen tal y como se trabajó en los simulacros, esto para aclarar que el simulador es un escenario de preparación centrado en la práctica de los estudiantes, pero por más completo que sea no puede contemplar todas las variables y situaciones que se dan en la realidad laboral. Además, la pretensión 
educativa es preparar a los estudiantes para que sean capaces de afrontar situaciones diversas, pero no se pueden abordar todas las particularidades que un ambiente real requieren.

\section{Estrategia de Aprendizaje}

A partir de las dificultades se inició el diseño de la estrategia pedagógica, la primera de ellas, la creación de espacios de práctica se consideró la aplicación de metodologías activas de aprendizaje que permitieran evidenciar la adquisición de la competencia por parte del estudiante; en otras palabras, el propósito es que el individuo pueda demostrar en el desempeño de su tarea, un desarrollo efectivo o superior en un trabajo o situación, lo que implicaría que la competencia es profunda y permanentemente asociada en sus comportamientos profesionales que lo lleven a una actuación apropiada ante la situación dada. (Sena y Diaz, 2013).

Para un efecto más impactante en la apropiación de los estudiantes se empleó el aprendizaje situado donde el aprender y hacer son acciones inseparables. Este aprendizaje destaca la importancia de la actividad y el contexto, reconoce que es un proceso de enculturación en el cual los estudiantes se integran gradualmente a una comunidad. En consecuencia, los alumnos deben aprender en el contexto. (Diaz, 2003), por lo tanto, se recrean diversos ambientes laborales, para seleccionarlos se hizo un análisis previo donde se tuvieran empresas de diversos tipos porque esto afecta significativamente el análisis, además, porque confronta al estudiante con diversos escenarios que pueda encontrar en su vida laboral.

Desde esa perspectiva de aprendizaje situado se estructuraron para el programa algunas estrategias que permiten que los estudiantes mejoren sus procesos teórico-prácticos. La primera estrategia es el diseño, planificación, construcción y publicación de un laboratorio virtual en el que se trabajará el área higiene y seguridad industrial que permite hacer el diagnóstico de las condiciones de trabajo basado en la identificación de peligros y valoración de riesgos de diversos ambientes laborales. Según Barrios y Marín (2013) "Los laboratorios virtuales son una solución actual para la creciente necesidad de que los alumnos puedan realizar prácticas desde su hogar". Además, de que el laboratorio puede ser útil como herramienta de refuerzo y apoyo para potenciar 
los conocimientos o como elemento didáctico en las aulas para fomentar un entorno participativo y constructivista. Además, potencia la adquisición de competencias (Molina, 2012).

La siguiente estrategia consiste en la creación de un Consultorio de la Gestión de la Seguridad y Salud Laboral para Pymes, con el que se pretende ser un centro de atención para las pequeñas y medianas empresas para abordar temáticas relacionadas al programa. El estudiante del consultorio debe diagnosticar, planear y ejecutar acciones para la gestión de los riesgos laborales. Para la adecuada ejecución de la propuesta la Institución va a diseñar y producir un software que ayude a los estudiantes a auditar el cumplimiento mínimo de los requisitos establecidos para la implementación del SGSST y crear el plan de intervención para la mitigación del riesgo. Esta estrategia está en fase de diseño e inicio del desarrollo.

La otra estrategia es modificar el módulo de práctica profesional acorde con las herramientas de simulación que se van a implementar, para que así la Institución tenga conocimiento de las áreas de desempeño específicas en el campo real y pueda estructurar o reorientar el programa con miras a responder a las necesidades del entorno laboral. Esta estrategia está planteada, penas se ha iniciado el desarrollo. Se mencionan en el documento porque el proyecto obedece a una estrategia integral de mejoramiento para el programa, no de acciones aisladas o la mera implementación de un software.

El proceso inicial comenzó como un laboratorio online. Los weblabs se dividen en dos categorías: Laboratorios Virtuales y Laboratorios Remotos. En el primero, la interfaz del usuario trabaja contra la simulación de un proceso. En el segundo, el alumno desde una ubicación remota accede al proceso desarrollado sobre una planta real o en maqueta, interactuando con el mismo de forma tal que le es posible variar los parámetros de control, ejecutar experimentos alternativos, observar los resultados obtenidos y eventualmente descargarlos de la web (Masanet, Zavalla, Fernández, 2011). El proyecto realizado corresponde a la primera clase, donde el estudiante accede en línea, encuentra una empresa y debe identificar los riesgos y realizar todo el análisis y proponer un plan de intervención para mediar dichos riesgos encontrados.

Una crítica fuerte de los laboratorios online es no tienen en cuenta las ideas de los estudiantes durante el desarrollo de la práctica, porque ya vienen diseñados los experimentos desde la programación (Vásquez, 2009). Otra complejidad detectada es que en algunos casos los laboratorios contienen 
errores conceptuales debido a que algunos programadores no cuentan con los conocimientos en la disciplina. No todos los laboratorios virtuales presentan un ambiente intuitivo, por lo que exigen conocimientos previos por parte de los estudiantes en el manejo de herramientas T.I.C. (Vásquez, 2009).

Para evitar este tipo de falencias el proyecto de realización se hace con expertos que han trabajado el tema, cada uno de ellos cuenta con un asesoramiento en diseño instruccional, orientación en la elaboración de guiones, el acompañamiento de un equipo de asesores pedagógicos que realizan la integración con lo que van a trabajar los ingenieros, quienes van revisando lo que se plantea desde lo pedagógico sea tecnológicamente posible. Además, cuenta con un grupo de diseñadores que manejan toda la parte gráfica. El autor o experto es guiado acompañado desde que inicia la idea hasta que se convierte en un producto palpable y hace las pruebas de verificación.

De igual manera, la programación se realizó de tal forma, que no hay dos casos iguales de análisis para el estudiante, para ello se generan diversas empresas, diferentes riesgos y diferentes soluciones para que dependa más de las decisiones tomadas por el estudiante que de una respuesta concreta. Para tomar en cuenta las decisiones de los estudiantes, el laboratorio llega hasta el análisis y evaluación de los peligros y la detección de los riesgos, sin embargo, los controles y las estrategias de mitigación están fuera de la herramienta, pero hacen parte de la experiencia de aprendizaje, es decir, el trabajo en el laboratorio le da los insumos a los estudiantes para que ellos propongan como producto final su plan de mitigación del riesgo para la empresa, por eso se refiere a una experiencia de aprendizaje inmersivo, donde todo está articulado para que no sólo sea la interacción con la herramienta, sino que depende de todo un proceso de apropiación por parte del estudiante.

Por tal razón, para darle una mayor versatilidad a la herramienta de software el proceso fue mutando para dar como resultado una combinación entre laboratorio y simulador, con características de los dos, como se expondrá más adelante.

La idea del simulador nace como resultado de un análisis previo en el que se identifican varias necesidades, la primera de ellas es que cada entorno laboral tiene unas particularidades que modifican de manera significativa el análisis, tanto que en la indagación se encontró simuladores únicamente centrados en empresas de construcción o en empresas de químicos. Por lo tanto, se realizó una indagación de los posibles sectores empresariales que estuvieran 
presentes en el contexto laboral de los estudiantes que permitieran diversos tipos de análisis en la cuestión de riesgos laborales. Al finalizar el proceso se llegó a cinco tipos de empresas (laboratorio, manufactura, oficina, pozo petrolero, servicios generales de aseo); transversales para el análisis de riesgos biológicos, físicos, químicos, naturales, de seguridad y biomecánicos. De esa manera, el estudiante puede simular en diversos tipos de organización y cumplir el objetivo primordial de aprendizaje que consiste en que el alumno sea capaz de analizar los riesgos laborales en cualquier tipo de empresa, porque la estrategia no se centra en un ambiente laboral, se centra en la apropiación de una metodología de análisis que el estudiante pueda aplicar en cualquier empresa.

La estructuración de la herramienta se hace a través de la metodología LEGO, por medio de la cual se trabaja de manera modular con la posibilidad de agregar o quitar piezas, reutilizar y minimizar costos cuando requiera actualizar los recursos y los personajes. La reutilización podría tener lugar dentro de un lapso de varios años. En la lógica de la reutilización el tiempo es crucial, se pueden adherir componentes rehusados en lugar de desarrollarlos por completo (Hsuan y Hansen, 2007). Esto permite que si a futuro se desea agregar otra empresa al análisis se puede implementar reutilizando lo que ya se tiene, para ello la metodología de desarrollo debe pensarse de esa manera. También si se requiere la implementación de nuevos peligros u otras modificaciones dadas por el cambio de metodología de análisis o de normas.

La estructuración de la estrategia de aprendizaje se diseñó de tal forma que abarque lo disciplinar y a su vez fuese transversal a todos los riesgos, por lo tanto se hizo un estudio de las diferentes metodologías o herramientas de evaluación como la GTC- 45, el Biogaval, la Gatiso de Benceno y el análisis de colores; todas coinciden en los siguientes pasos: identificación del riesgo, diagnóstico de la situación, evaluación de los peligros, análisis, a partir de este la mitigación que es la propuesta de intervención para disminuir los factores de riesgo. Teniendo claras estas premisas se va a desarrollar un proceso que sirva de práctica y experiencia a través del cual el estudiante trabajará en cada una de las etapas hasta proponer la intervención.

La identificación del riesgo cubre la visualización de la empresa, de los puestos o áreas de trabajo, el estudiante debe identificar de manera visual y con las ayudas pertinentes los peligros que existen. Luego tiene una información de la situación que se la dan o los directivos de la empresa o sus colaboradores. 
El diagnóstico define los posibles peligros y se evalúan según los métodos de evaluación dados para cada riesgo. De acuerdo con esa evaluación permite proponer diferentes estrategias para mitigar ya sean en la fuente, en el medio o en el colaborador.

El simulador cubrirá dos momentos de experiencia: la individual, en la cual el estudiante encontrará un escenario de simulación guiado y autónomo para que realice prácticas y afiance la identificación de un escenario y diagnostique el riesgo. Se habla de simulación autónoma porque el alumno simulará y enviará los resultados, y la retroalimentación será generada por el simulador. La grupal que brinda al equipo un escenario, propuesto de manera aleatoria por la herramienta, con las mismas condiciones de simulación para el grupo. En la segunda experiencia la simulación no es guiada, el estudiante realizará todo el proceso sin orientaciones, deberá aplicar lo visto en el módulo, consignará la información en fichas, definirá el tipo de riesgo, creará el plan de intervención y se lo enviará al tutor a través del aula.

La estrategia está aplicada para los siguientes módulos de la manera que se explica en la tabla 1.

Tabla 1. Módulos con el simulador de riesgos laborales.

\begin{tabular}{|l|c|l|l|}
\hline Módulo & Semestre & Riesgo laboral & Unidades que abarca \\
\hline Higiene y seguridad industrial 2 & 4 & Riesgo biológico & Unidad 1 y 2 \\
\hline Higiene y seguridad industrial 2 & 4 & Riesgo químico & Unidad 3 y 4 \\
\hline Higiene y seguridad 3 & 5 & Riesgo físico & Unidad 1, 2, 3 y 4 \\
\hline Higiene y seguridad 4 & 6 & Riesgo de seguridad & Unidad 1 y 2 \\
\hline Higiene y seguridad 4 & 6 & Riesgo natural & Unidad 3 y 4 \\
\hline Ergonomía & 4 & Riesgo ergonómico & Unidad 1, 2, 3 y 4 \\
\hline
\end{tabular}

Fuente: Creación propia.

\section{Modelo para la simulación}

Aunque el sistema contemple etapas similares en todos los riesgos, el modelo de simulación es completamente diferente. 
Riesgo biológico: para el riesgo biológico, en el área individual, se trabaja con la GTC-45, y recrea una oficina la cual tiene cinco personajes, el simulador elige aleatoriamente tres, y tiene siete peligros y elige de manera aleatoria a cinco. Entonces cuenta con una aleatoriedad de 525 casos diferentes para que cada estudiante tenga una experiencia diferente. Esta simulación es autónoma y la herramienta retroalimenta al estudiante de acuerdo con sus respuestas e incluye peligros que no son biológicos para que sea capaz de identificarlos.

Una vez finaliza la simulación individual el alumno procede a la grupal. Esta simulación no es autocontenida, a cada equipo se le asigna el mismo caso y cada uno debe simular para después reunirse a consolidar y confrontar la información que van a consignar en el plan de intervención final. Esta simulación difiere en algunos aspectos de la individual debido a que trabaja con la metodología Biogaval y por ende cuenta con algunos escenarios propios de la metodología. La herramienta suministra los resultados de la evaluación y los alumnos establecen los posibles controles.

Riesgo Químico: el funcionamiento para el riesgo químico es similar que el biológico, se cuenta con 5 empresas, 7 riesgos, 5 personajes y son elegidos de la misma forma de aleatoriedad, cambian obviamente los riesgos, ya no se trata de agentes biológicos sino de sustancias químicas según como lo define la GTC-45. Asimismo, la metodología de evaluación del riesgo se hace con la Gatiso de Benceno para la parte grupal.

Riesgo de Seguridad: el funcionamiento para el riesgo de seguridad es similar al biológico puesto que cuenta con cinco empresas, siete riesgos y cinco personajes que son elegidos de la misma forma de aleatoriedad. Además, cambian los riesgos de manera que ya no se trata de agentes biológicos o sustancias químicas, sino de riesgos de seguridad de los colaboradores según como lo define la GTC-45. Asimismo, la metodología de evaluación del riesgo se hace con la GTC-45.

Estos tres riesgos se verán a profundidad en el capítulo 1.

Riesgo Biomecánico o ergonómico: En el riesgo biomecánico o ergonómico se trabajan cuatro riesgos fundamentales que son: higiene postural, corrección de posturas, manejo de cargas laborales, biopsicosocial y diseño de puestos de trabajo. Aquí se trabajan tres metodologías de evaluación que son REBA, 
RULA y OWAS, de igual manera, sigue los procedimientos para el manejo de cargas. El simulador tiene cuatro personajes y cada uno de ellos incluye cuatro posturas. Este riesgo opera para las cinco empresas ya definidas, lo que arroja inicialmente 75 posibilidades de análisis, como esta simulación es únicamente grupal, se espera que a cada grupo se le origine un caso diferente. Cada personaje tiene unas características antropométricas diferentes para que el análisis de un personaje a otro sea completamente diferente. Dependiendo el riesgo que se trabaje el escenario de simulación cambia drásticamente, hay escenarios donde el estudiante debe elegir el mejor inmobiliario para la salud postural del colaborador, en otros debe cambiar los grados de las posturas hasta llegar a la ideal, en otras debe escuchar a los colaboradores y proponer planes de mejora, en otros debe cambiarle y sugerirle los elementos de protección personal (EPP) u otros implementos para una buena postura en el trabajo.

Este riesgo se tratará en profundidad en el capítulo 2.

Riesgo natural: el riesgo natural se hace para establecer un plan de emergencia, de manera que el reto educativo para el estudiante es crearle a esa organización dicho plan. Por lo tanto, se tienen las cinco empresas para las cuales se crearon cinco escenarios de riesgo: uno muy factible a la inundación, otro al vendaval, uno más al terremoto, otro al deslizamiento, y así sucesivamente hasta cubrir todos los riesgos naturales que da la GTC-45. El simulador combina aleatoriamente las cinco empresas con los cinco ambientes, generando así 25 casos de estudio diferentes. El estudiante debe identificar en la empresa que se le asigna cuáles son los riesgos más eminentes y hacer la evaluación por medio de la metodología de colores. También contempla los riesgos isotrópicos y los sociales, ampliando el análisis. Con la evaluación del riesgo natural el estudiante propone el plan de emergencia, que incluye las rutas de evacuación, los puntos de encuentro, la ubicación de los extintores conforma las brigadas. En otras palabras, elabora todo el Plan de emergencias para dicha organización, al cambiar los factores de riesgo en el ambiente cambia todo el análisis.

Este riesgo se tratará en profundidad en el capítulo 3.

Riesgo físico: para este riesgo se construyeron planos con los que el estudiante ingresa a cada área de la empresa, toma mediciones que están programadas en rangos de acuerdo con los instrumentos de medida que se relacionan 
así: temperatura (termómetro), iluminación (luxómetro), ruido (sonómetro), vibración (vibrómetro), radiación (dosímetro). De esa manera, se cubren los peligros físicos que determina la GTC-45. De acuerdo con el resultado de la medición el estudiante determinará si hay un peligro o no y de allí inicia la evaluación con la metodología expuesta en la GTC - 45. Durante la evaluación diligencia la matriz de riesgos, saca la prioridad de estos y establece el plan de controles.

Este riesgo se tratará en profundidad en el capítulo 4.

\section{Desarrollo de la experiencia de aprendizaje}

Como se puede evidenciar cada riesgo y cada empresa tiene sus particularidades, para todos los riesgos se conservaron las empresas, vale la pena aclarar que las empresas son del mismo sector, pero no son las mismas, para cada riesgo estas varían y por eso se hizo un trabajo con cada experto para definir la estrategia de aprendizaje a emplear. A partir de dichas estrategias se trabajaron desde lo pedagógico las competencias que debería apropiar el estudiante con la simulación, se establecieron los resultados que arrojaría el simulador y cuáles los trabajos o estrategias de aprendizaje para los estudiantes, como se explicará en cada uno de los capítulos.

Se crearon los mapas de funcionalidad, descomponiendo la competencia en el saber, hacer y ser, y obteniendo las tareas (qué debería hacer dentro de la estrategia de aprendizaje) estudiante, simulador y tutor. Con estos mapas se hizo la evaluación para el desarrollo tecnológico, para proceder a los guiones. A continuación, un inventario de guiones para que los lectores dimensionen la magnitud del proyecto, en riesgo biológico para cada empresa el guion es cercano a las 75 páginas, para cinco empresas son 325 páginas, que incluyen las fichas técnicas de los personajes, los diálogos, los textos que van en las pantallas, las acciones de los botones, los comentarios de diseño y programación. El riesgo químico de cada uno es de 80 páginas y de seguridad de 75 . En riesgo ergonómico cada guion por empresa es de 300 páginas. El riesgo natural se hizo un solo guion de 100 páginas y el riesgo físico cada guion constaba de 60 páginas.

Esto significa que solamente en guiones se realizaron 3050 páginas para todas las empresas y todos los riesgos. Estos riesgos se revisaron y ajustaron, por 
ejemplo, para riesgo físico se integraron las cinco empresas en un guion de 100 páginas. Con los ajustes se completaron 1500 páginas aproximadamente, esto debido al reajuste de algunos asuntos de programación, se integraron escenarios que se duplicaban y se hizo un modelo de guion para cada riesgo. Superado lo anterior, se unificó la información que era transversal, de tal manera que la reducción de páginas fue significativa. Este proceso duró diez meses.

Uno de los procesos más complejos para el desarrollo y el diseño, fue traducir a lenguaje de programación más de 1500 páginas. Solamente los diálogos de los personajes ocupan más de 130 páginas, más de 100 animaciones en 2D y, además, se construyeron cincuenta escenarios en 3D para riesgo natural, físico y ergonómico. Por eso, un proyecto de esta magnitud debe obedecer a una apuesta institucional encaminada a producir un verdadero cambio educativo.

Las interfaces se crearon de tal manera que hubiese una línea de diseño en cada riesgo, para ello se identificó cada uno de ellos con un color respectivo, manteniendo una línea visual y tipográfica, para que cada simulador sirva para un módulo diferente. La imagen 1. Gama de colores para cada riesgo.
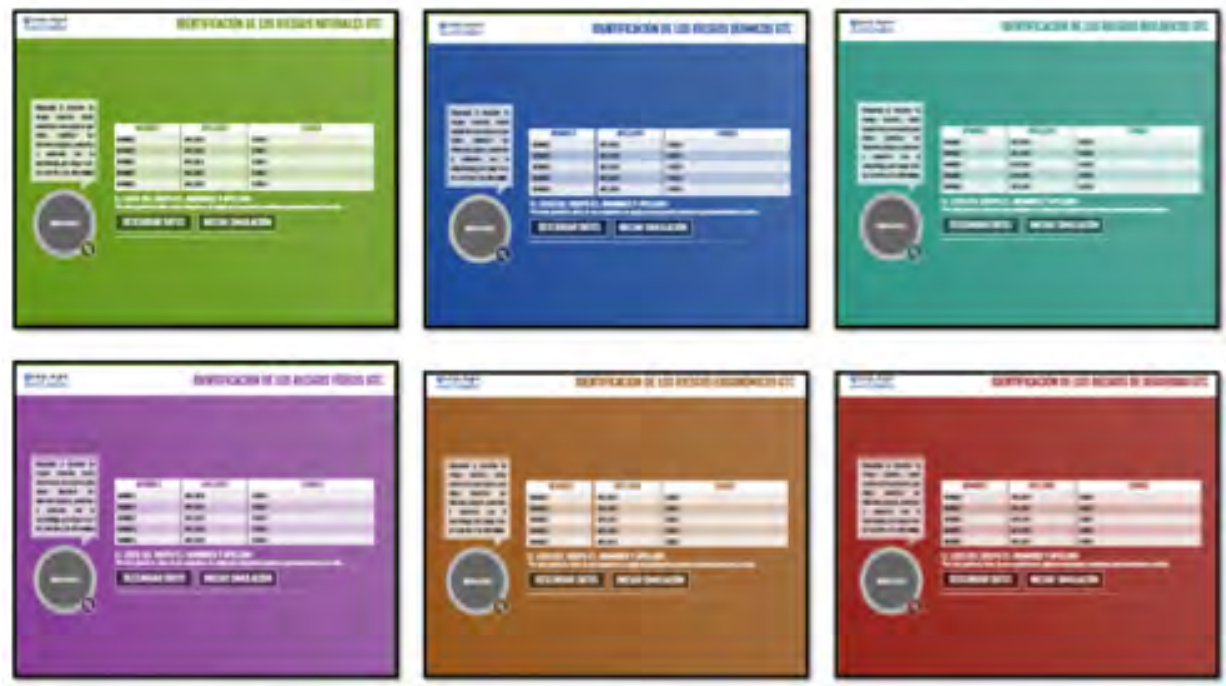

Figura 1. Gama de colores para cada simulador.

Fuente: Creación propia. Politécnico Grancolombiano y Picolab (2017) 
También se creó a un personaje de ayuda al estudiante que se llamó Bernard, en honor a Bernardino Pazinni, el cual es transversal a todos los riesgos y todas las empresas porque su finalidad es orientar al estudiante en cada uno de los escenarios, darle indicaciones y ayudarle en el proceso de simulación. Todo tiene una finalidad, el personaje no es humano porque se quieren resaltar los elementos de protección personal como los guantes, las gafas, el casco, las botas.

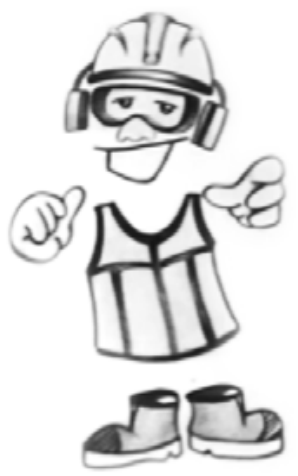

A. Boceto
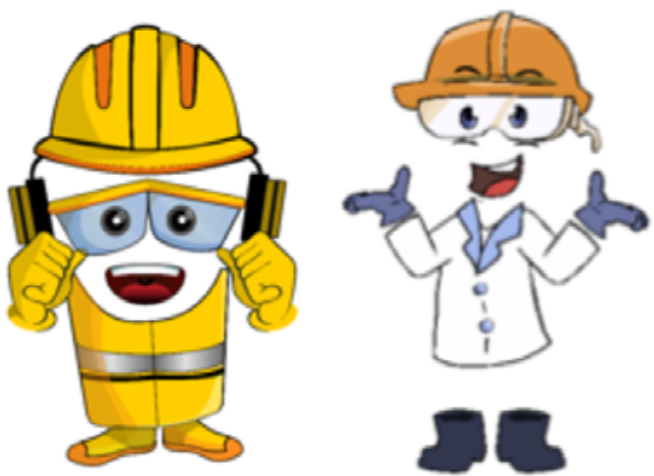

B. Primera versión
C. Versión final

Figura 2. Versiones de Bernard.

Fuente: Creación propia. Politécnico Grancolombiano y Picolab (2017)

Cada riesgotiene sus propios personajes, para Biológico, Químicoy de Seguridad se hicieron cinco personajes, que se mantienen en las empresas, pero cada uno tiene otros cargos y están expuestos a diferentes peligros y cuentan con un supervisor que presenta cada empresa, es decir, cinco personajes más. En Ergonomía se crearon cuatro personajes a los cuales les cambian las posturas. En Físico se tienen cinco gerentes, uno para cada empresa. En Natural se cuenta con un gerente para cada empresa y proporciona toda la información. En conclusión, significa que se crearon aproximadamente treinta personajes diferentes. 

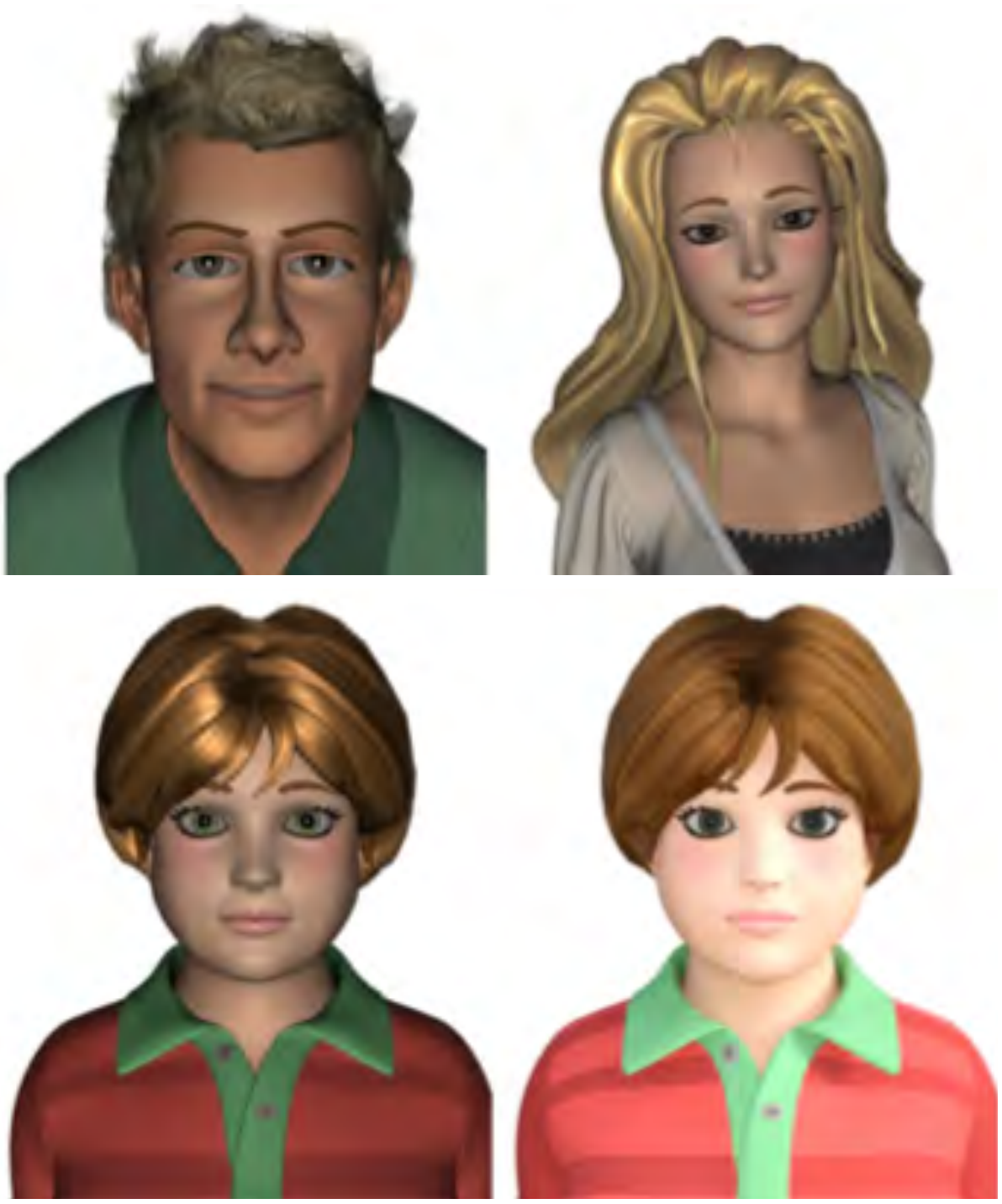

Figura 3. Bosquejo de los personajes para los diferentes riesgos.

Fuente: Creación propia. Politécnico Grancolombiano y Picolab (2017)

Antes de la programación se realizaron los Mock Ups que muestra la diagramación de los escenarios para su aprobación e iniciar la programación. Ver la figura 4. 


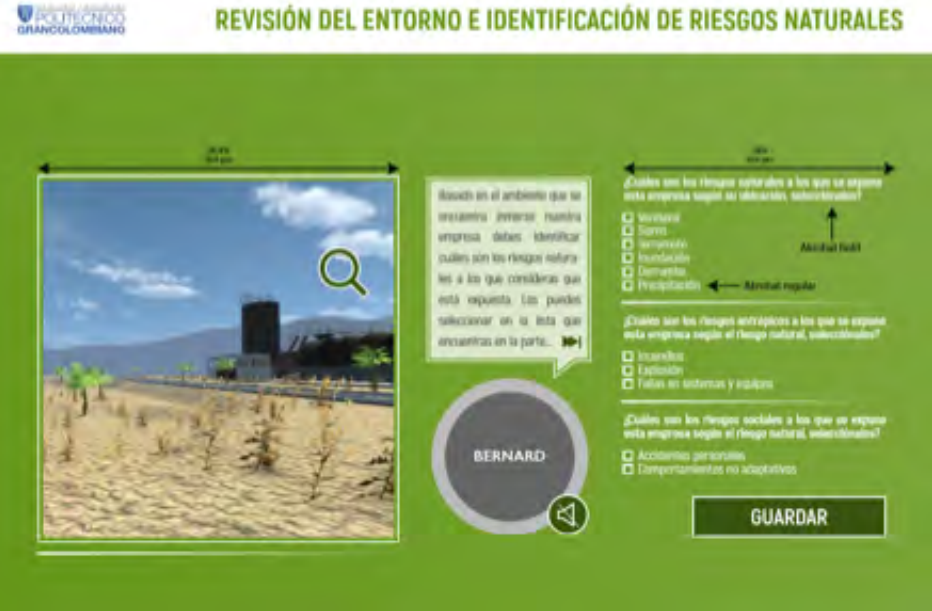

Figura 4. Mock up de riesgo natural.

Fuente: Creación propia. Politécnico Grancolombiano y Picolab (2017)

Los escenarios de 3D permiten trabajar, aumentar la sensación de realidad y generan mayor inmersión del alumno con la herramienta. Esta sensación es única. Según Vera, Ortega y Burgos (2003) los experimentos realizados por investigadores han demostrado que la curva de aprendizaje con realidad virtual es más rápida y consigue una mayor y mejor asimilación, debido a que los estudiantes utilizan casi todos sus sentidos en el proceso de aprendizaje. Bajo esa premisa se crearon diversos escenarios 3D según las necesidades educativas manifestadas por los expertos en los guiones como lo muestra la figura 5.

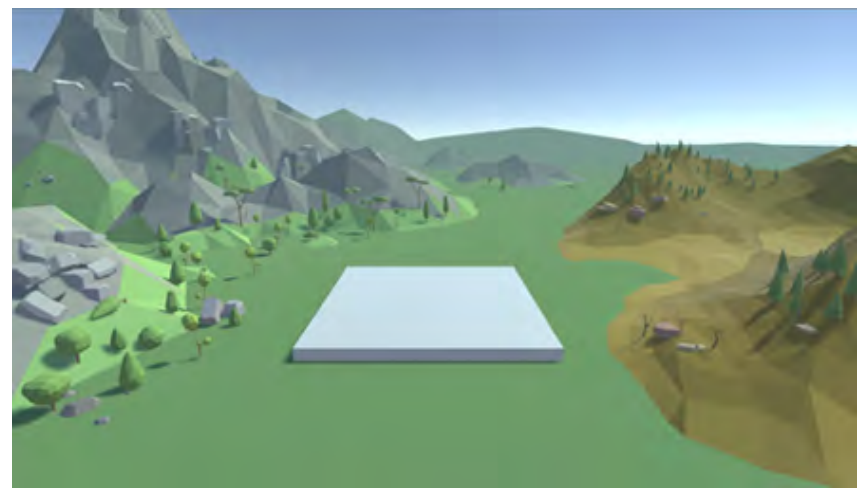

Figura 5. Escenario 3D - Riesgo Natural - Derrumbe.

Fuente: Creación propia. Politécnico Grancolombiano y Picolab (2017) 
La programación se hizo en el software Unity que permite la combinación de los escenarios 3D con los requerimientos de programación y la inclusión de las fórmulas propias de cada método para cada riesgo. Para la realización del diseño gráfico, las animaciones 2D y 3D, y la programación del software de acuerdo con los guiones fue contratada la empresa Picolab.

\section{Resultados obtenidos}

En este proceso se obtuvo una herramienta de simulación, tal vez la más completa en riesgos laborales, diferenciándose de todas las indagadas, en que la mayoría sólo cubren un máximo de tres, y esta cubre los seis tipos de riesgos laborales.

Se hace un proceso completo para el análisis de los riesgos laborales en diferentes ambientes y cubre todos los pasos que van desde la identificación de los peligros, el diagnóstico, análisis, evaluación y mitigación. Además, permite una variedad de peligros de diversa índole dentro de cada riesgo complejizando el proceso y haciéndolo más significativo para el estudiante.

Tal vez el resultado más positivo para la Institución es contar ahora con un equipo de docentes capaces de producir herramientas de simulación para futuros proyectos o para mejora de los actuales, ya que el proceso implica ver el aprendizaje desde una perspectiva de aplicación donde se evidencie lo aprendido y es muy diferente a una perspectiva de conceptualización donde el estudiante debe apropiar conceptos que debe posteriormente aplicar.

\section{Conclusiones}

Como este capítulo es una invitación a conocer todo el proceso que se hizo en cada riesgo no se presentan conclusiones, en cambio se hace una exhortación a que usted lea cada uno de los capítulos y saque sus propias conclusiones.

\section{Bibliografía o referencias}

Amaya, G. (2009). Laboratorios reales versus laboratorios virtuales, en la enseñanza de la física: Revista El hombre y la máquina. (Vol. XXI, Núm. 33. pp. 82-95.) Universidad Autónoma de Occidente. Cali, Colombia. 
Barrios T. y Marín M. (2013) Motivación en la enseñanza a través de laboratorios virtuales Universidad Tecnológica Nacional Resistencia. Foro Mundial de Educación en Ingeniería. Ponencia, Cartagena.

Díaz Barriga Arceo, F. (2003). Cognición situada y estrategias para el aprendizaje significativo. REDIE. Revista Electrónica de Investigación Educativa, 5 (2), 105-117. Recuperado en: http://www.redalyc.org/articulo.oa?id=15550207

Pascuas Rengifo, Y., \& Bocanegra García, J., \& Ortiz Lozada, E., \& Pérez Castillo, J. (2012). Desarrollo dirigido por modelos para la creación de laboratorios virtuales. Scientia Et Technica, XVII (51), 119-125.

Hsuan J. y Hansen P. (2007) Platform development: implications for portfolio management. Gest. Prod., São Carlos, v. 14, n. 3, p. 453-461, São Paulo. Recuperado en: http://www.scielo.br/pdf/gp/v14n3/a03v14n3

Icontec Internacional (2012). Guía Técnica Colombiana para la identificación de los Peligros y la valoración de los riesgos en seguridad y salud ocupacional. Editada por el Instituto Colombiano de Normas Técnicas y Certificación (ICONTEC). Bogotá. Recuperada en: http://idrd.gov.co/sitio/idrd/sites/default/files/imagenes/gtc450. pdf

Masanet M., Zavalla E., Fernández A. (2011). Un enfoque integrado para las prácticas de laboratorio en la educación a distancia. TE\&ET | Revista lberoamericana de Tecnología en Educación y Educación en Tecnología, Número 06. Instituto de Automática, Facultad de Ingeniería, Universidad Nacional de San Juan, San Juan

Molina, J. (2012). Herramientas virtuales: Laboratorios virtuales para ciencias experimentales, una experiencia con la herramienta VCL. En: X Jornades de xarxes d'investigació en docència universitària. Universidad de Alicante, Alicante. Recuperado de: https://web.ua.es/en/ice/jornadas-redes-2012/documentos/ posters/245405.pdf

Serna H. y Diaz A. (2013) Metodologías activas del aprendizaje. Editorial Fundación Universitaria María Cano, Bogotá. Recuperado de: http://portal.fumc.edu.co/ publicaciones/libros/metod/metodologias.pdf

Tobón S. (2004) Formación Basada en Competencias. Ecoe Ediciones. Bogotá. Citado en: Pérez, N., \& Amaya, D., \& Ramos, O. (2006). Laboratorio virtual en la formación por competencias. Ciencia e Ingeniería Neogranadina, 16 (2), 113-116. Universidad Nueva Granada, Bogotá. Recuperado en: http://www.redalyc.org/articulo. oa?id=91116212

Vásquez C. (2009) “Laboratorios virtuales". (Núm. 20). En: García H. (2016) Uso de los laboratorios virtuales para la enseñanza-aprendizaje del concepto materia y sus 
propiedades en estudiantes de grado noveno. Tesis de Maestría Universidad Nacional de Colombia. Manizales. Recuperado de: http://www.bdigital.unal.edu. co/56591/1/10130019.2016.pdf

Vera G, Ortega J. A. y Burgos M. (2003) La realidad virtual y sus posibilidades Didácticas. Revista Ética-net. Año 2. Vol 2. Universidad de Granada; Granada. Recuperado en: http://www.ugr.es/ sevimeco/revistaeticanet/Numero2/Articulos/Realidadvirtual. pdf

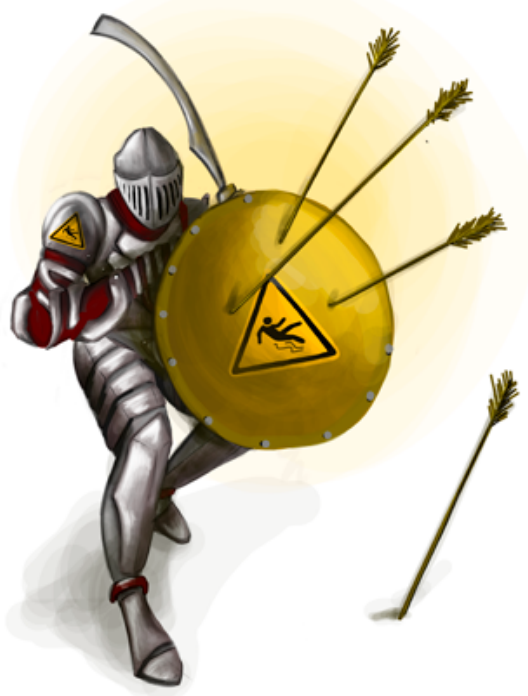

